\title{
Environmental factors predicting nephritis in systemic lupus erythematosus
}

\author{
Tim McAlindon, Lena Giannotta, Nick Taub, David D’Cruz, Graham Hughes
}

\begin{abstract}
Objectives-To evaluate social class, ethnic origin, and various endocrine variables as potential risk factors in the development of nephritis in patients with systemic lupus erythematosus (SLE).

Methods-A cross-sectional survey was carried out of all outpatients with SLE attending the lupus Clinic of St Thomas's Hospital from March to October 1992 using retrospective survival data. The main outcome measure was the duration of SLE before the onset of nephritis.

Results-Two hundred and ninety six women and 11 men were studied; the male patients were excluded from the analysis. Univariate analysis showed an increased risk of nephritis in patients with SLE of West Indian origin with $54 v 19 \%$ with nephritis at five years, in patients of lower social class, in patients who did not drink alcohol, and in those with a history of fetal loss after the onset of lupus. No significant effect of the age of onset of SLE, use of oral contraceptives, normal pregnancy, or smoking was seen. Multivariate analysis showed that ethnic origin did not influence the risk of nephritis independently of social class.
\end{abstract}

Conclusions-Factors associated with socioeconomic deprivation may increase disease severity in patients with SLE.

(Ann Rheum Dis 1993; 52: 720-724)

Systemic lupus erythematosus (SLE) is a multisystem autoimmune disease of unknown aetiology with an estimated prevalence of $15-51$ cases per $100000 .^{1}$ Renal disease is common in this disorder, occurring in at least $30 \%$ of affected subjects. ${ }^{2}$ Patients with SLE with nephritis are an important subgroup because they have increased morbidity and mortality, require additional treatment, and consume more health care resources..$^{3-5}$

The identification of the factors which increase the susceptibility of patients with SLE to the development of nephritis has the potential to increase our understanding of the pathogenesis of this complication and to enhance the overall management of patients with lupus. Research indicates an increased susceptibility to nephritis in lupus patients of black racial origin, in those with certain HLA gene phenotypes and, in some studies, those with a younger age of onset..$^{5-8}$ Of these, racial influences appear to have the strongest effect.
It is therefore of great interest that the racial differences in the incidence and severity of SLE (which are not confined to the USA ${ }^{9}$ ) do not appear to have a genetic basis, at least with respect to the major histocompatibility complex. ${ }^{1011}$ Additional evidence for environmental influences on the disease expression of SLE is provided by studies showing important confounding effects of medical insurance status (as a proxy for socioeconomic class) on the development of renal failure and on overall survival. ${ }^{5}{ }^{12}$ Further work is therefore needed to unravel the contributions of these variables to the development of nephritis in SLE and to establish whether other differences exist between those who develop nephritis and those who do not. We therefore investigated the roles of racial origin, social class, and other potential risk factors in a multiracial patient group attending a National Health Service lupus clinic at St Thomas's Hospital, London, United Kingdom.

\section{Patients and methods}

SUBJECTS

Subjects studied here consisted of all patients with SLE attending the lupus clinic at St Thomas's Hospital during 1992. For inclusion in the study subjects were required to fulfil four or more of the revised American Rheumatism Association criteria for the classification of SLE. ${ }^{13}$ The date of onset of SLE was ascertained retrospectively by a structured interview in association with case note review. Subjects in whom this was unclear were excluded from the study.

Because of the possibility of biases resulting from not including information about subjects who had died within the study period, we also attempted to obtain data from the case notes of all 10 patients who had died during the five years before the study began, during which time details had been systematically recorded by one of us (DD'C).

\section{NEPHRITIS}

Patients were classified as having nephritis on the basis of either (a) a positive renal biopsy sample or $(b)$ persistent proteinuria (defined as greater than $0.5 \mathrm{~g} /$ day or greater than $3+$ if not measured) accompanied by an active urinary sediment with cellular casts or reduced creatinine clearance, or both.

The onset of nephritis was dated at the time of first observation of persistent proteinuria or urinary cellular casts. In the absence of 
documented proteinuria or casts the first abnormal creatinine clearance result was used.

PUTATIVE RISK FACTORS FOR NEPHRITIS IN SLE Demographic variables collected included ethnic origin, marital status, occupation or husband's occupation at the time of diagnosis, as well as cigarette and alcohol consumption subsequent to diagnosis. Subjects were allotted a social class according to occupation using the British standard occupational classification, but combining subgroups IIIa and IIIb because of small numbers. ${ }^{14}$ Endocrine data included use of oral contraceptives, number of pregnancies culminating in a live birth, and number of pregnancies resulting in fetal loss, before and after the diagnosis of SLE.

\section{STATISTICAL METHODS}

Statistical analysis was based on a survival time analysis from the diagnosis of SLE to the onset of nephritis using the log-rank test for univariate analysis. Proportional hazards regression (Cox survival analysis) was used to control for potential confounders. ${ }^{15}$ Forwards stepwise and backwards stepwise procedures were used to select those variables significantly associated with survival time at the 5\% level. To allow for any possible effect of left censorship in this analysis, it was then repeated with the inclusion of all available data relating to subjects who had died within the previous five years.

\section{ETHICS}

Approval from the St Thomas's Hospital ethical committee was obtained (ref. EC92/ 339).

\section{Results}

A total of 307 patients participated in the study, of whom most (296) were women. Their characteristics are presented in detail in tables 1 and 2 . Eighty one patients ( 75 women) had nephritis. Because of the small number of male patients $(11 ; 4 \%)$ and the possibility that the disease has different characteristics in men and women, the men were excluded from further analysis. In addition, we examined the case notes of the 10 subjects who had died in the five year period before the study and derived

Table 1 Characteristics of the female patients with systemic lupus erythematosus studied

\begin{tabular}{lcc}
\hline & $\begin{array}{l}\text { No nephritis } \\
(n=221)\end{array}$ & $\begin{array}{c}\text { With nephritis } \\
(n=75)\end{array}$ \\
\hline Ethnic origin & & \\
White & 186 & 58 \\
West Indian & 16 & 11 \\
Other & 19 & 6 \\
Social class & & 5 \\
I & 34 & 35 \\
II & 84 & 25 \\
III & 92 & 9 \\
IV+ (interquartile range) age (years) & 11 & $38(30-46)$ \\
Median (in (interquartile range) disease duration (years) & $41(32-48)$ & $8(5-13)$ \\
\hline
\end{tabular}

${ }^{\star}$ One subject was omitted as social class not known. sufficient data for further analysis from five of these (table 3).

Univariate survival analysis showed the effects of race $(p=0.002)$ and social class $(p=0.047$ ) (figs 1 and 2). Racial differences were most marked when comparing white patients with those of West Indian ethnic origin-for example, $17 \%$ of white patients had nephritis at five years ( $95 \%$ confidence interval (CI) 12 to $22 \%$ ) compared with $53 \%$ of patients of West Indian origin (95\% CI 25 to $80 \%$ ). Fetal loss after the onset of SLE was also associated with an increased risk of nephritis $(p=0.005)$, as was total abstinence from alcohol $(p=0.020)$. No significant effects were observed from the use of oral contraceptives before or after the onset of lupus, pregnancy with a normal outcome before or after the onset of lupus, or from smoking ( $p>0.05$ ).

To control for potential confounders, these variables, together with ethnic origin, social class, use of oral contraceptives, pregnancies, and age of onset of SLE were entered into forwards and backwards stepwise multivariable analyses using proportional hazards regression. Variables found to have significant independent associations $(p<0.05)$ with the development of lupus nephritis were ethnic origin (forwards stepwise selection), social class at diagnosis (backwards stepwise selection), alcohol consumption, and fetal loss after the onset of SLE. Table 4 shows the joint proportional hazards regression model for these variables adjusting for pregnancies and the use of oral contraceptives after the onset of SLE. In this final model social class appeared to be strongly related to nephritis, showing a gradient of increased risk with reduced social class and a greater estimate of risk than that from West Indian ethnic origin (hazards ratio 4.7 for social class IV with respect to I

Table 2 Overall incidence of nephritis according to ethnic origin and social class (one subject was omitted as social class was not known)

\begin{tabular}{llcr}
\hline Ethnic group & Social class & $\begin{array}{l}\text { No of } \\
\text { subjects }\end{array}$ & $\begin{array}{l}\text { No (\%) with } \\
\text { nephritis }\end{array}$ \\
\hline White & I & 34 & $4(12)$ \\
& II & 102 & $30(29)$ \\
West Indian & III (a + b) & 93 & $18(19)$ \\
& IV & 15 & $6(40)$ \\
& II & 0 & $-150)$ \\
Other & III (a + b) & 8 & $6(35)$ \\
& IV+ & 2 & $1(50)$ \\
& I & 5 & $1(20)$ \\
& II & 9 & $1(11)$ \\
& III $(\mathrm{a}+\mathrm{b})$ & 7 & $1(14)$ \\
\hline
\end{tabular}

Table 3 Characteristics of five of the 10 patients who died in the five years before the study for whom adequate data were available. None of the patients drank alcohol and all had nephritis

\begin{tabular}{llll}
\hline $\begin{array}{l}\text { Year of } \\
\text { death }\end{array}$ & $\begin{array}{l}\text { Racial } \\
\text { origin }\end{array}$ & $\begin{array}{l}\text { Social } \\
\text { class }\end{array}$ & $\begin{array}{l}\text { No of } \\
\text { miscarriages }\end{array}$ \\
\hline 1987 & White & IIIa & 4 \\
1988 & West Indian & NK & 0 \\
1988 & Asian & I & 0 \\
1989 & African & IIIa & 0 \\
1989 & White & IIIb & 0 \\
\hline
\end{tabular}

${ }^{\star} \mathrm{NK}=$ Not known. 


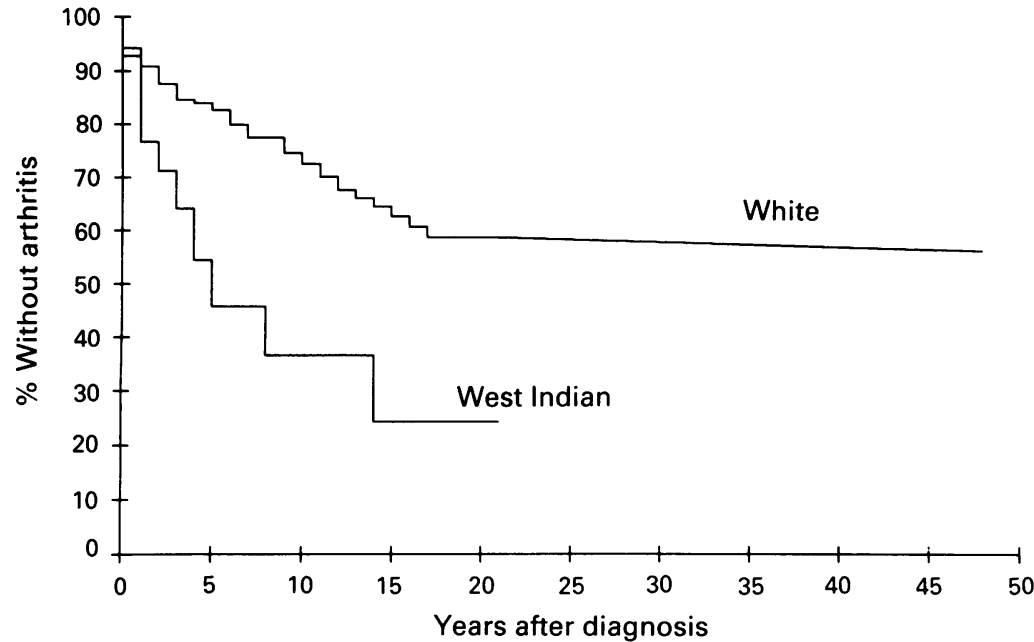

Figure 1 Development of nephritis in patients with systemic lupus erythematosus: the influence of ethnic origin.

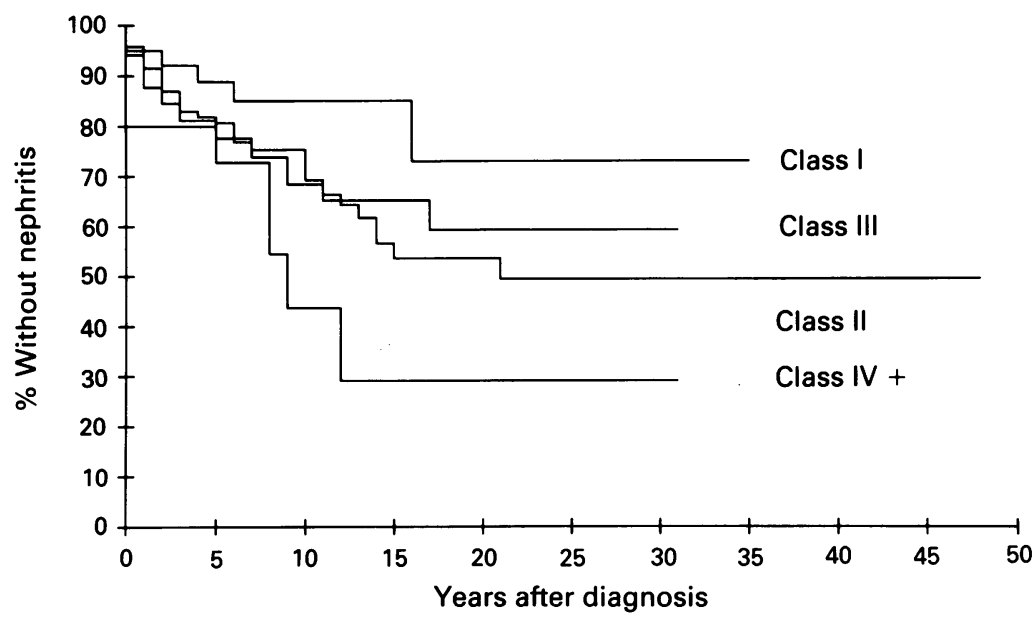

Figure 2 Development of nephritis in patients with systemic lupus erythematosus: the influence of social class.

Table 4 Results of proportional hazards regression analysis. Hazards ratios and p values for social class are relative to class $I$, and for ethnic origin are relative to white subjects. The overall $p$ value for social class was 0.035 and for ethnic origin was $0 \cdot 173$. One subject was omitted as social class was not known

\begin{tabular}{lclll}
\hline & $\begin{array}{l}\text { No of } \\
\text { patients }\end{array}$ & $\begin{array}{l}\text { Hazards } \\
\text { ratio }\end{array}$ & $\begin{array}{l}\text { 95\% Confidence } \\
\text { limits }\end{array}$ & p Value \\
\hline Social class & & & & \\
$\quad$ I & 39 & 1.0 & 1.1 to 7.2 & 0.035 \\
II & 119 & $2 \cdot 8$ & 1.0 to 6.9 & 0.060 \\
III (a + b) & 117 & 2.6 & 1.5 to 14.3 & 0.007 \\
IV+ & 20 & 4.7 & & \\
Ethnic origin & 244 & 1.0 & 1.0 to 3.9 & 0.059 \\
$\quad$ White & 27 & 1.9 & 0.3 to 2.1 & 0.727 \\
$\quad$ West Indian & 24 & 0.8 & 0.3 to 0.9 & 0.021 \\
$\quad$ Other & 145 & 0.6 & 1.3 to 3.6 & 0.004 \\
Alcohol consumption & 51 & 2.1 & 0.5 to 1.5 & 0.657 \\
Miscarriage after onset of SLE & 82 & 0.9 & 0.3 to 1.2 & 0.166 \\
Pregnancy after onset of SLE & 56 & 0.6 & & \\
Use of oral contraceptives & & & &
\end{tabular}

SLE $=$ Systemic lupus erythematosus.

$(\mathrm{p}=0.007)$ compared with 1.9 for West Indian $v$ white subjects $(p=0.059))$. No significant influence was observed from the use of oral contraceptives or from pregnancy after the onset of lupus.

To investigate the possibility of a left censorship effect in these data we then included the variables relating to five subjects who had died before the study began and repeated the univariate and multivariate analyses. With the exception of strengthening the level of significance of the effect of alcohol abstinence (from $p=0.021$ to 0.012 ), this made no material difference to the results.

\section{Discussion}

This study shows that patients with SLE of West Indian ethnic origin are at greatly increased risk of renal disease and suggests that much of this may be due to factors associated with low socioeconomic status. Studies in the USA have indicated that medical insurance status (used as a proxy for socioeconomic class) influences survival and the prevalence of renal failure in patients with SLE. ${ }^{5}$ Because of this we were interested to determine if ethnic origin and socioeconomic status retained independent effects in a population to whom access to health care was freely available.

The emergence of social class as the variable with the strongest independent influence on the risk of nephritis (hazards ratio $4 \cdot 7$ for class $\mathrm{IV}+v$ class $\mathrm{I}$ ) in patients with SLE raises a number of important issues. Systemic lupus erythematosus remains a disease of unknown cause and it is possible that subjects in lower socioeconomic groups are somehow preferentially exposed to the putative aetiological agent. A more likely explanation, however, is that other factors associated with reduced social class are responsible for increased disease severity in these subjects. The influence of medical insurance status on outcome in patients with SLE in the USA provides some evidence to suggest that suboptimal health care might be one mechanism for this. ${ }^{57}$ There are, however, other mechanisms in which social class may influence disease severity. Poverty and unemployment, for example, have been linked to increased illness and mortality. ${ }^{16}$ Levels of education might also be important and, in particular, ignorance of symptoms indicating SLE may lead to delayed presentation and treatment. An important implication of this is that early intervention and treatment may influence outcome in SLE.

The results of the joint proportional hazards regression suggest that West Indian ethnic origin has no independent effect on the risk of nephritis; however, numbers are small and West Indian ethnicity is not represented in social class I. Clearly there is close interplay between these two variables, and as ethnic origin emerged as a predictor of nephritis on the forwards regression model it is possible that both make independent contributions to the overall risk. Previous investigators have inferred a direct influence of race on the clinical and laboratory manifestations of SLE from the differences in these parameters found between black and white populations, ${ }^{7}$ though clear HLA associations with SLE in black subjects have not been shown. ${ }^{11}$

Two other factors associated with increased risk of nepthritis were fetal loss and abstinence from alcohol. Miscarriages are a recognised phenomenon in $\mathrm{SLE}^{13}$ and were associated with anticardiolipin antibodies in 10/53 (19\%) patients. As neither the use of oral contracep- 
tives nor the occurrence of pregnancies with favourable outcome was associated with any increase in the risk of nephritis, it is likely that fetal loss is a marker for more severe disease. The prognostic value of laboratory measures in SLE, including anticardiolipin antibodies, is currently being investigated.

Why alcohol abstinence should be associated with nephritis is unclear, and we considered the possibility that this association had arisen spuriously as a result of left censorship of cohort data. At least $50 \%$ of the SLE deaths occurring in the five years before this study occurred in patients who did not drink alcohol and all of these had nephritis. It is possible that a moderate intake of alcohol confers a degree of protection as a result of cardiovascular effects. On the other hand, although subjects were asked about their intake subsequent to the onset of SLE, it is likely that responses were biased towards current consumption and it is possible that these subjects had stopped drinking alcohol as a result of more active or severe disease.

The lack of any effect from the use of oral contraceptives or from normal pregnancy on the risk of nephritis is reassuring and of relevance in counselling women with SLE. Endocrine factors have traditionally been thought to be intrinsic in the pathogenesis of SLE $^{1718}$ but it is noteworthy that, as here, other studies have not shown adverse effects from oral contraceptives or pregnancy. ${ }^{19} 20$ Similarly, the age of onset of SLE was not shown in this study to influence the risk of nephritis.

The main limitations of this study result from (a) referral bias, (b) its cross sectional/ retrospective design, and (c) its application to women only. The study group was of relatively high social class (median II) and comprised relatively few non-white patients. These factors would reduce the chance of detecting the effects of class and ethnic origin on the risk of nephritis. Also, the median social class was similar in the nephritis and non-nephritis groups, although the former comprised a greater percentage of non-white patients. The number of non-white, non-West Indian subjects was too small for separate analysis and it was not possible to determine whether their risk was similar to white patients or to those of West Indian origin; nor was it possible to determine whether the duration of stay in the United Kingdom had any influence in the West Indian subgroup.

The mode of recruitment of subjects into this study also generates a potential for left censorship by not taking into account data from subjects who died during the period referred to in the questionnaire. We estimate a less than $10 \%$ attrition rate in terms of patients lost to follow up; however, this remains an estimate because good diagnostic records were not kept. On the other hand, systematic records were kept of all 10 deaths of patients with SLE during the five years before the study and we took the opportunity to examine their case notes and managed to obtain relevant data from five of these. The characteristics of these patients broadly corresponded with the study findings and their inclusion in the analysis made no material difference to the results. Furthermore, most of the findings here agree with the results of previous work and it is notable that five of the 10 subjects with SLE who had died before the study did not drink alcohol. ${ }^{1-5}$ Nevertheless, as these five do not represent all of the deaths during the period referred to in the questionnaire, a left censorship effect cannot be entirely ruled out.

Social class was assessed using the standard British classification system which reflects the nature of occupation as well as income in six broad categories. ${ }^{14}$ It is occupation oriented and therefore may be a crude measure when applied to a group of women. Moreover, it provides little information about important related variables such as levels of education. As this was determined at the time of onset of SLE it also does not take into account any changes in status which may have occurred during the course of the disease.

Direct influences of comorbidity were not taken into account in this study. West Indian populations may be at increased risk of hypertension and diabetes mellitus, and it is possible that these influence the onset of nephritis. As current hypertension may reflect lupus nephritis any assessment of attributable risk would have to be made on the basis of an earlier blood pressure measurement, which we were unable to determine in this retrospective study.

In conclusion, we have shown independent associations of social class, fetal loss, and alcohol abstinence with nephritis in SLE. The influence of social class suggests that factors associated with deprivation somehow influence disease severity in SLE. These require urgent investigation.

We thank the Erycinus Charitable Trust for its financial support and Sue Chinn, reader in medical statistics, department of public health medicine, United Medical and Dental Schools of public health medicine, United Medical and Dental Scho
Guy's and St Thomas's Hospitals, for statistical advice.

1 Hochberg M C. Systemic lupus erythematosus. Rheum Dis Clin North Am 1990; 3: 617-39.

2 Nossent J C, Bronsveld W, Swaak A J. Systemic lupus erythematosus III. Observations on clinical renal involvement and follow-up of renal function: Dutch experience with 110 patients followed prospectively. $A n n$ Rheum Dis 1989; 48: 810-6.

3 Ginzler E M, Diamond H S, Weiner M, et al. A multicenter study of outcome in systemic lupus erythematosus I. Entry variables as predictors of prognosis. Arthritis Rheum Entry variables as

4 Rosner S, Diamond H S, Weiner M, et al. A multicenter study of outcome in systemic lupus erythematosus. II. study of outcome in systemic lupus erythematosu

5 Studenski S, Allen N B, Caldwell D S, Rice J R, Polisson R P. Survival in systemic lupus erythematosus. Arthritis Rheum 1987; 12: 1326-32.

6 Hochberg M C, Boyd R E, Ahearn J M, et al. Systemic lupus erythematosus: a review of clinico-laboratory features and immunogenetic markers in 150 patients with emphasis on demographic subsets. Medicine (Baltimore) 1985; 64 285-95.

7 Ward M M, Studenski S. Clinical manifestations of systemic lupus erythematosus: identification of racial and socioeconomic influences. Arch Intern Med 1990; 150 849-53.

8 Fronek Z, Timmerman L A, Alper C A, et al. Major histocompatibility complex associations with systemic lupus erythematosus. Am f Med 1988; 85: 42-4.

9 Samanta A, Roy S, Feehally J, Symmons D P M. The prevalence of diagnosed SLE in whites and Indian Asian immigrants in Leicester city, UK. Br $\mathcal{J}$ Rheumatol 1992; 31: 679-82.

10 Hochberg M C, Fronek Z, Timmerman L A, et al. Major histocompatibility complex associations with systemic lupus erythematosus. Am $\mathcal{F}$ Med 1988; 85: 42-4. 
11 Reveille J D, Schrohenloher R E, Acton R T, et al. DNA analysis of HLA-DR and DQ genes in American blacks with SLE. Arthritis Rheum 1989; 32: 1243-7.

12 Ward M M, Studenski S. Clinical manifestations of systemic lupus erythematosus: identification of racial and socioeconomic influences. Arch Intern Med 1990; 150: 849-53.

13 Tan E M, Cohen A S, Fries J F, et al. The 1982 revised criteria for the classification of SLE. Arthritis Rheum 1982; 25: 1271-7.

14 Standard occupational classification. Vol 3. London: HMSO, 1991.

15 Dixon W J. BMDP statistical software manual. California: University of California Press, 1990: 769-82.
16 Smith R. "Without work all life goes rotten". BMF 1992; 305: 972 .

17 Masi A T, Kaslow R A. Sex effects in SLE: a clue to pathogenesis. Arthritis Rheum 1978; 21: 480-4

18 Lahita R G. Sex steroids and the rheumatic diseases. Arthritis Rheum 1985; 28: 121-6.

19 Lockshin M D, Reinitz E, Druzin M L, Murrman M, Este D. Lupus pregnancy: case-control prospective study demonstrating absence of lupus exacerbation during or after pregnancy. $A m$ f Med 1984; 77: 893-8.

20 Julkunen $\mathrm{H} \mathrm{A}$. Oral contraceptives in systemic lupus erythematosus: side effects and influence on the activity of SLE. Scand 7 Rheumatol 1991; 20: 427-33. 